\title{
Proteínas PE/PPE: potenciais alvos na prospecção de novos fármacos para o tratamento da tuberculose - Breve revisão
}

\author{
PE/PPE proteins: potential targets in prospecting new drugs for the treatments \\ of tuberculosis - Brief review
}

Edriele da Silva Pinto ${ }^{1}$; Rita Terezinha de Oliveira Carneiro ${ }^{2 *}$
${ }^{1}$ Faculdade Maria Milza - FAMAM, Governador Mangabeira - Bahia, Brasil, 44350-000, edrielesilva4554@hotmail.com; https://orcid.org/0000-0002-2349-9348

2*Faculdade Maria Milza - FAMAM, Governador Mangabeira - Bahia, Brasil, 44350-000, ritaterezinha@gmail.com; https://orcid.org/0000-0002-7568-6487 (autor correspondente)

\section{Resumo}

A tuberculose (TB) é a principal causa de óbito por infecção bacteriana em todo o mundo. $\mathrm{O}$ uso irracional dos fármacos anti-TB contribui para aquisição de resistência em linhagens de Mycobacterium tuberculosis (Mtb) e não se tem registro de novos fármacos anti-TB nas últimas duas décadas. Proteínas PE/PPE são fundamentais no processo infeccioso e no metabolismo energético do Mtb, portanto se caracterizam como alvos de novos fármacos. O objetivo foi analisar dados disponíveis sobre as estruturas das proteínas PE/PPE de Mtb. Trata-se de uma revisão integrativa, e a metodologia consistiu em busca e análise de trabalhos completos publicados no período de 1998 a 2018. Foram analisados 23 artigos. Proteínas PE/PPE apresentam alta solubilidade em compostos polares dificultando sua análise. A bioinformática atua como importante ferramenta na elucidação estrutural e funcional das proteínas; bem como na descrição das interações fármaco-proteína, minimizando os efeitos adversos dos ensaios clínicos e o uso de animais em pesquisa. A bioinformática é de grande valia na prospecção de novos antibióticos especialmente nesse contex to que se registra aumento dos casos de infecção resistente. Acreditamos que a robustez de dados obtidos pela técnica de criomicroscopia possa garantir a análise mais completa das proteínas PE/PPE.

Palavras-chave: Epítopo; Proteômica; Resistência antimicrobiana. 


\begin{abstract}
Tuberculosis (TB) is leading cause of death from bacterial infection worldwide. Irrational use of anti-TB drugs contributes to acquisition of resistance in Mycobacterium tuberculosis (Mtb) strains and there has been no record of new anti-TB drugs in the last two decades. PE/PPE proteins are fundamental in the infectious process and in the energy metabolism of Mtb, therefore they are characterized as targets of new drugs. The objective was to analyze available data on the structures of Mtb PE/PPE proteins. It is an integrative review, the methodology consisted of searching and analyzing complete papers, published from 1998 to 2018. Twenty three articles were applicable. PE/PPE proteins have high solubility in polar compounds, making their analysis difficult. Bioinformatics acts as an important tool in the elucidation and structural function of proteins; as well as in the description of drug-protein interactions, minimizing the adverse effects of clinical trials and the use of animals in research. Bioinformatics is of great value in the search for new antibiotics, especially in this context where there is an increase in cases of resistant infection. We believe that the robustness of data obtained by the cryomicroscopy technique guarantees the most complete analysis of PE/PPE proteins.
\end{abstract}

Keywords: Antimicrobial resistance; Epitope; Proteomics.

\title{
1. Introduçãa
}

A tuberculose (TB) configura-se como a principal causa de óbito por infecção bacteriana de único agente em todo o mundo. Em 2018 foram registradas 1,4 milhões mortes exclusivamente por TB e a notificação de 10,4 milhões de novos casos da doença (WHO, 2019). A TB é passível de cura obtida por meio da adesão ao tratamento, que no Brasil é ofertado gratuitamente pelas Uunidades Básica de Saúde - UBS (BRASIL, 2019).

O uso irracional dos fármacos anti-TB estabelece condições favoráveis para a evolução das linhagens de Mtb, que se tornam resistentes aos antibióticos empregados na terapêutica da doença (ALIPANAH et al., 2018). O fenômeno de resistência aos fármacos anti-TB é fator complicador no enfretamento da TB pois prolonga, onera e dificulta seu controle e tratamento, e portanto é considerado como um urgente problema de saúde pública em escala mundial. O último relatório da Organização Mundial da Saúde (OMS) sobre TB revela ocorrência de 484 mil casos de TB resistente em todo o mundo; recomenda medidas que minimizem o surgimento de novos casos e o correto tratamento daqueles que já existem (WHO, 2019).

Dentre as medidas mitigadoras para o tratamento das formas clínicas de TB resistentes 
adota-se tratamentos com tempo menor de duração e a inclusão de fármacos mais agressivos como a clofazimina e bedaquilina, que ainda aguardam liberação para uso em território nacional (SILVA et al., 2020). O registro de produção dos antibióticos remetem a década de 80, revelando a necessidade e urgência na prospecção de novos fármacos anti-TB (STURKENBOOM et al., 2018).

Os programas de bioinformática atuam como importantes ferramentas na elucidação de novos alvos terapêuticos, haja vista sua capacidade de produzir dados a respeito das interações fármaco-proteína com rapidez e confiança, minimizando o uso de modelo animal nas experimentações, bem como o aparecimento dos efeitos adversos que ocasionalmente são relatados nos ensaios clínicos conduzidos em humanos (NUNES, 2015). Nessa perspectiva, análise da estrutura e função de proteínas centrais $(h u b)$ no metabolismo e/ou transmissão de microrganismos são requeridas e incentivadas (PITASSI et al., 2014).

A família gênica PE/PPE (proline-glutamic acid e proline-proline-glutamic acid, respectivamente) codificam as proteínas transmembranas no $\mathrm{Mtb}$, as quais desempenham importante papeis durante etapas da infecção e ao longo do curso da TB (PHERLAN et al., 2016). Dada a sua importância para a fisiologia e para o metabolismo do Mtb, as proteínas PE/PPE podem atuar como eventuais alvos moleculares dos novos tratamentos anti-TB (LANGERMANS et al., 2005).

\section{Material e Métodos}

Trata-se de uma revisão integrativa da literatura sobre a estrutura das proteínas PE/PPE sintetizadas pelo Mtb. A coleta de dados foi realizada por meio de buscas por artigos publicados em bases como: PubMed, Scielo, LILACS, no período de 1998 a 2018. Os descritores utilizados foram: "estrutura e função das proteínas PE/PPE"; "desenvolvimento de novos fármacos antiTB" e "mecanismo de ação dos fármacos anti-TB" nos idiomas português e inglês.

Os critérios de inclusão foram: $a$ ) fator de impacto/qualis do periódico; $b$ ) trabalhos completos. E os critérios de exclusão foram: $a$ ) publicações anteriores a 1998; $b$ ) material de divulgação elaborado por empresa farmacêutica/centro de pesquisa responsáveis por produzir ou distribuir fármacos anti-TB. Também foram utilizados manuais de controle e tratamento da 
TB elaborados por órgãos competentes.

A análise dos artigos selecionados seguiu a metodologia proposta por Minayo (2001), que se dividem em 3 etapas, a saber: análise prévia do conteúdo apresentados pelos autores (organização dos dados); exploração do conteúdo de cada artigo (leitura e confronto dos dados apresentados pelos autores) e tratamento dos dados (interpretação das informações obtidas).

\section{Resultados}

Inicialmente foram encontrados 100 artigos, mas apenas 23 se enquadraram nos critérios adotados. A leitura dos trabalhos supracitados possibilitou estabelecer 4 tópicos, aqui considerados como estruturantes no entendimento sobre a estrutura das PE/PPE, a saber: $i$ ) estrutura da família gênica PE/PPE; ii) atuação das proteínas PE/PPE no processo infeccioso e metabolismo do Mtb; iii) mecanismos dos fármacos anti-TB e iv) prospecção de fármacos antiTB com atuação sobre as PE/PPE (Tabela 1).

Tabela 1. Descrição dos tópicos estruturantes sobre a estrutura e atuação das proteínas PE/PPE produzidas por $M$. tuberculosis (Mtb) e do mecanismo de ação dos fármacos anti-TB já empregados no tratamento da doença.

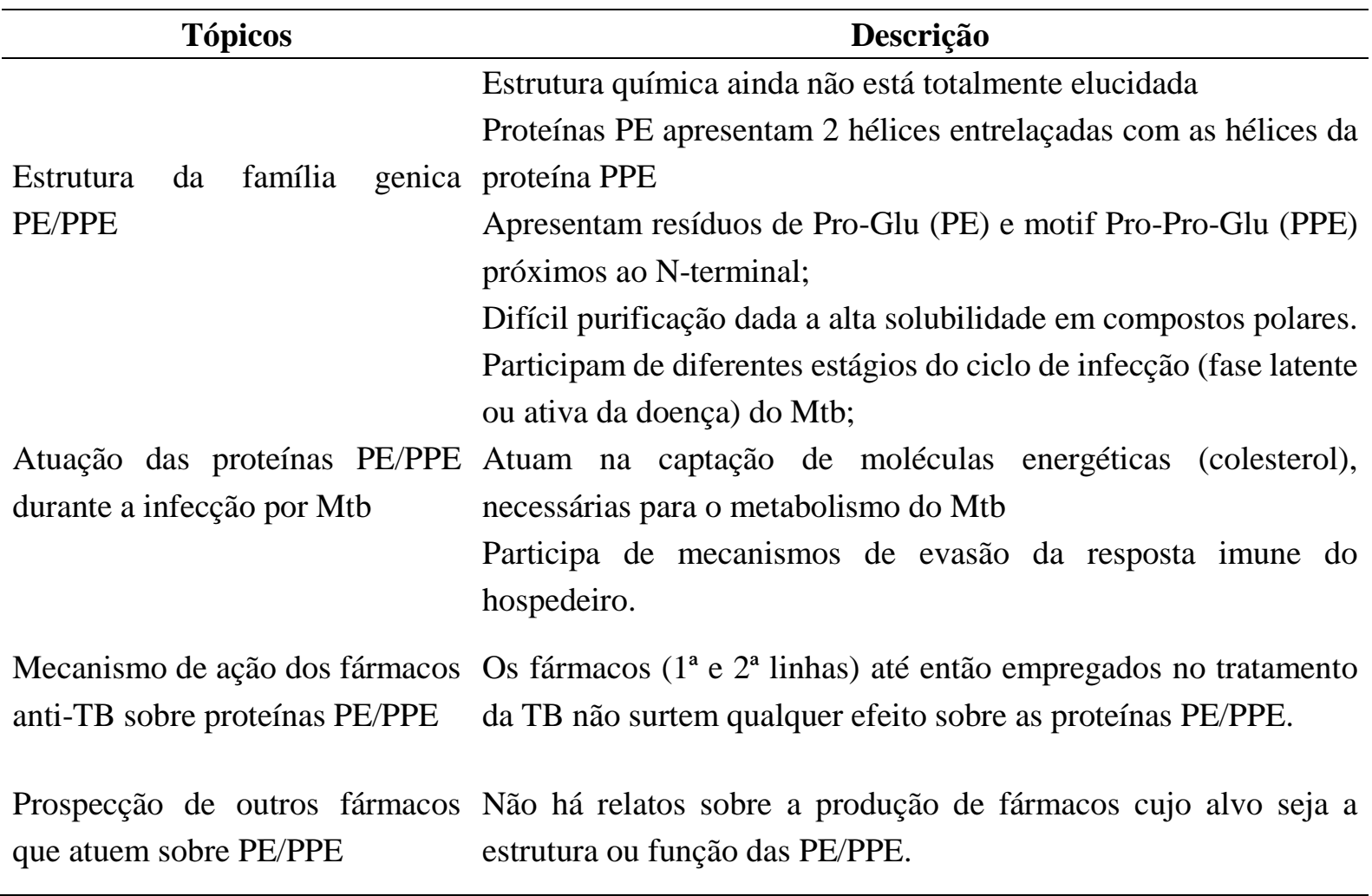




\section{Discussão}

A estrutura das proteínas PE/PPE foi descrita como um entrelaçado de hélices que se inserem na parede celular do Mtb e apresenta alta solubilidade em compostos polares (OVERBEEK et al., 1999; STRONG et al., 2006), como ilustra a Figura 1.
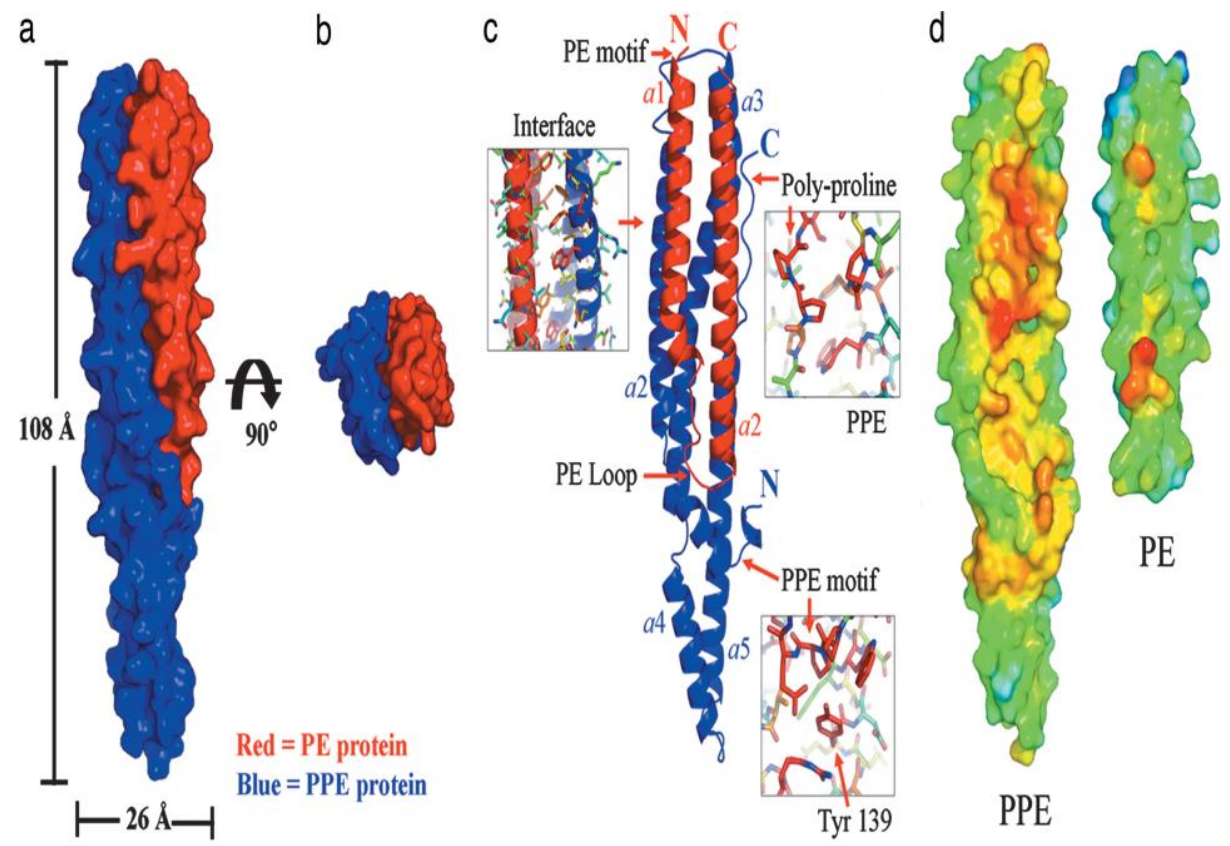

Figura 1. Estrutura das proteínas da família gênica PE/PPE. a - Estrutura tridimensional específica de PE em vermelho e PPE em azul; b - Vista superior das enzimas PE/PPE; c - A sequência motif das proteínas da família gênica PE/PPE e d - regiões de polaridade em amarelo e vermelho e de apolaridade em azul das proteínas PE/PPE. Fonte: STRONG et al, 2006.

As proteínas PE/PPE tem estruturas homologas entre si e são membros da família gênica que atuam na patogenia e virulência do Mtb (REED et al., 2009; WOODWORTH et al., 2016). As referidas proteínas são caracterizadas pela presença de sequência bem conservada e homóloga de aminoácidos no $N$-terminal, cujo domínio é evidenciado pela presença de pro-glu (prolina-glutamina) nas posições 8 e 9 das proteínas $\mathrm{PE}$, e pela presença de pro-pro-glu (prolinaprolina-glutamina) nas proteínas PPE (BRENNAN et al., 2001; GORDON et al., 2001).

Apesar de ainda não haver um consenso sobre o papel biológico das PE/PPE (RANAKRISHNAN, 2000; AKHTER et al., 2012; CHEN et al., 2017) dados de homologia evidenciam vantagens bastante significativas par ao Mtb proporcionadas por estas proteínas em 
momentos cruciais no processo infeccioso, especialmente durante o momento da invasão nas células hospedeiras (BRENNAN et al., 2001; GORDON et al., 2001).

Entre outas funções as PE/PPE também são capazes de induzir forte resposta imunológica no indivíduo hospedeiro (DELOGUE \& BRENNAN, 2001; CHAKHAIYAR et al., 2004; LI et al., 2004; KHAN et al., 2008); captam eficientemente as moléculas destinadas ao processo oxidativo do metabolismo energético do Mtb após a invasão na célula hospedeira (KHUBAID et al., 2016; AHMAD et al., 2018); participa do processo de aquisição de resistência aos fármacos anti-TB (KOHLI et al., 2012).

De modo geral as PE/PPE garantem condições de sobrevivência do Mtb apesar das condições hostis imposta pelas células durante a resposta de defesa do indivíduo infectado (PERDIGÃO et al., 2014; TUNDUP \& MOHAREER, 2014). Apesar das importantes atuações desempenhadas pelas proteínas PE/PPE nenhum dos fármacos já empregados na $1^{\mathrm{a}}$ e $2^{\mathrm{a}}$ linhas de tratamento anti-TB tem ação sobre a estrutura ou função dessas proteínas (DALCOMO, 2012; BRASIL, 2019). A Tabela 2 sumariza os fármacos e seus respectivos mecanismos de ação.

Tabela 2. Caracterização dos fármacos empregados no tratamento anti-TB com seus respectivos mecanismos de ação.

\begin{tabular}{lcl}
\hline \multicolumn{1}{c}{ GRUPOS } & FÁRMACO & \multicolumn{1}{c}{ MECANISMO DE AÇÃO } \\
\hline $\begin{array}{l}\text { Grupo 1: Medicamentos } \\
\text { orais de primeira linha }\end{array}$ & $\begin{array}{c}\text { isampicina (R), } \\
\text { etambutol (E), } \\
\text { pirazinamida (Z) }\end{array}$ & $\begin{array}{l}\text { Inibe a síntese do RNAm } \\
\text { Inibe a síntese do ácido micólico, } \\
\text { Inibe síntese do arabinogalactano } \\
\text { Inativa atividade enzimática de Mtb }\end{array}$ \\
$\begin{array}{l}\text { Grupo 2: Medicamentos } \\
\text { injetáveis }\end{array}$ & $\begin{array}{c}\text { canamicina (C), amicacina (Am), } \\
\text { capreomicina (Cap) }\end{array}$ & $\begin{array}{l}\text { Se ligam a subunidade ribossômica } \\
\text { inativando síntese proteica do Mtb }\end{array}$ \\
\hline $\begin{array}{l}\text { Grupo 3: } \\
\text { Fluoroquinolonas }\end{array}$ & $\begin{array}{c}\text { ofloxacino (Ofx), levofloxacino } \\
\text { (Lfx), moxiflaxacino (Mn) }\end{array}$ & $\begin{array}{l}\text { Bloqueia duplicação do } \\
\text { micobacteriano }\end{array}$ \\
\hline $\begin{array}{l}\text { Grupo 4: Medicamentos } \\
\text { orais bacteriostáticos 2 } \\
\text { linha }\end{array}$ & $\begin{array}{c}\text { etionaminda (Et); protionamida } \\
\text { cicloserina (Cs), teridizona (Trd) } \\
\text { Ácido p-amiosalicílico (PAS) }\end{array}$ & Inibe síntese de ácido micólico \\
\hline
\end{tabular}

FONTE: Adaptada de DALCOMO, 2012; BRASIL, 2019. 
Considerando que a TB é um grave problema de saúde pública e que o registro das formas resistentes da doença tem aumentado mundialmente (WHO, 2019), entende-se a urgência na prospecção de novas drogas destinadas ao combate da TB.

A produção de novos fármacos esbarra na complexidade inerente à sua síntese e pelo elevado custo para sua obtenção, mas especialmente devido as interações farmacocinéticas passíveis de ocorrer ao longo do processo. Todavia. a busca por novos fármacos conta com os avanços na química biológica que melhor descreve as interações metabólicas e fisiológicas das moléculas com seus respectivos alvos moléculas, e com a expressiva contribuição das ferramentas oferecidas pelos programas computacionais (NUNES, 2015).

Análises estruturais de proteínas centrais (chamadas de $h u b$ ) no metabolismo e/ou transmissão de microrganismos vem sendo amplamente analisadas por ferramentas de bioinformática e utilizadas na elucidação de novos fármacos (PITASSI et al., 2014). As atividades desempenhadas pelas proteínas PE/PPE possibilitam classificá-las como proteínas centrais na infecção e no metabolismo do Mtb, porém não existem, ou pelo menos não foram encontrados estudos destinados a prospecção de novos fármacos anti-TB destinados a alterar a estrutura ou interferir na atividade destas proteínas.

A maior dificuldade de estudar as proteínas PE/PPE consiste na sua alta solubilidade em compostos polares que restringe ou inviabiliza sua análise por técnicas convencionalmente utilizadas (STRONG et al, 2006). Uma alternativa para análise dessas proteínas pode ser o emprego da criomicroscopia eletrônica, técnica que possibilita analisar as proteínas em diferentes estados conformacionais de maneira robustas em comparação as demais metodologias analíticas descritas até o momento (VILAS et al., 2018).

\section{Perspectivas futuras}

Os avanços na oferta da internet mais veloz e com capacidade aumentada de geração e importação de dados (internet 5G); as melhorias nas ferramentas dos programas de bioinformática e a aplicação de técnicas de microscopia eletrônica de altíssima resolução contribuem para a prospecção de novos fármacos e na discriminação de seus potenciais alvos moleculares. Esperamos que toda a tecnologia disponível possa ser utilizada na elucidação de 
tratamentos mais eficientes contra doenças como a TB.

\section{Referencias}

AKHTER, Y.; EHEBAUER, M. T.; MAKHOPADHYAY, S. et al. (2012). The PE/PPE multigene family codes for virulence factors and is a possible source of mycobacterial antigenic variation: Perhaps more? Biochimie. v. 94, n. 1, p. 110-116. https://doi.org/10.1016/j.biochi.2011.09.026

ALIPANAH, N.; JARLSBERG, L.; MILLER, C. et al. (2018). Adherence interventions and outcomes of tuberculosis treatment: A systematic review and meta-analysis of trials and observational studies. PLos Med, v. 15, n. 7. https://doi.org/10.1371/journal.pmed.1002595

BRASIL. (2019). Ministério da Saúde. Secretaria de Vigilância em Saúde. Departamento de Vigilância Epidemiológica. Manual de recomendações para o controle da tuberculose no Brasil. 2a edição atualizada. Brasília-DF.

BRENNAN, M. J.; DELOGU, G.; BARDAROV, S. et al. (2001). Evidence that mycobacterial PE_PGRS proteins are cell surface constituents that influence interactions with other cells. Infection And Immunity. v. 69, n. 12, p. 7326-7333. https://doi.org/10.1128/IAI.69.12.7326$\underline{7333.2001}$

CHEN, X.; CHENG, H-F.; ZHOU, J. et al. (2017). Structural basis of the PE-PPE protein interaction in Mycobacterium tuberculosis. Journal of Biological Chemistry, v. 292, n. 41, p. 16880-16890. https://doi.org/10.1074/jbc.M117.802645

CHAKHAIYAR, P.; NAGALAKSMI, T.; ARUNA, B. et al. (2004). Regions of high antigenicity within the hypothetical PPE major polymorphic tandem repeat open-reading frame, Rv2608, show a differential humoral response and a low $\mathrm{T}$ cell response in various categories of patients with tuberculosis. The Journal Of Infectious Diseases. v. 190, n. 7, p. 1237-1244. https://doi.org/10.1086/423938

DALCOMO, M. M. P. (2012). Tratamento da tuberculose sensível e resistente. Revista Pulmão, v. 21, p. $65-77$.

DELOGU, G.; BRENNAN, M. J. (2001). Comparative immune response to PE and PE_PGRS antigens of Mycobacterium tuberculosis. Infection And Immunity. v. 69, n. 9, p. 5606-5611. https://doi.org/10.1128/iai.69.9.5606-5611.2001

GORDON, S.; EIGLMEIER, K.; GARNIER, T. et al. (2001). Genomics of Mycobacterium bovis. Tuberculosis. v. 81, n. 1-2, p. 157-163. https://doi.org/10.1073/pnas.1130426100

KHAN, N.; ALAM, K.; NAIR, S. et al. (2008). Association of strong immune responses to PPE protein Rv1168c with active tuberculosis. Clinical And Vaccine Immunology. v. 15, n. 6, p. $974-$ 980. https://doi.org/10.1128/CVI.00485-07 
KOHLI, S.; SINGH, Y.; SHARMA, K. et al. (2012). Comparative genomic and proteomic analyses of PE/PPE multigene family of Mycobacterium tuberculosis H37Rv and H37Ra reveal novel and interesting differences with implications in virulence. Nucleic Acids Research. v. 40, n. 15, p. 7113-7122. https://doi.org/10.1093/nar/gks465

KHUBAIB, M.; SHEIKH, J. A.; PANDEY, S. et al. (2016). Mycobacterium tuberculosis cooperonic PE32/PPE65 proteins alter host immune responses by hampering Th1 response. Frontiers In Microbiology. v. 7, n. 17, p. 1-8. https://doi.org/10.3389/fmicb.2016.00719

LANGERMANS, J. A. M.; DOHERTY, T. M.; VERVENNE, R. A. W. et al. (2005). Protection of macaques against Mycobacterium tuberculosis infection by a subunit vaccine based on a fusion protein of antigen 85B and ESAT-6. Vaccine. v.23, n. 21, p. 2740-2750. https://doi.org/10.1016/j.vaccine.2004.11.051

LI, Y.; MILTNER, E.; WU, M. et al. (2004). A Mycobacterium avium PPE gene is associated with the ability of the bacterium to grow in macrophages and virulence in mice. Cellular Microbiology. v. 7, n. 4, p. 539-548. https://doi.org/10.1111/j.1462-5822.2004.00484.x

MINAYO, M. C. L. (2001). Pesquisa social: teoria, método e criatividade. 19a edição. Editora Vozes, Petropólis-RJ.

NUNES, I. K. C. (2015). A importância do estudo de metabolismo nos estágios iniciais de desenvolvimento de fármacos. Revista Virtual de Química. v. 7, n. 2, p. 649-662. https://doi.org/10.5935/1984-6835.20150028

OVERBEEK, R.; FONSTEIN, M.; D'SOUZA, M. et al. (1999). The use of gene clusters to infer functional coupling. PNAS. v. 96, n. 6, p. 2896-2901. https://doi.org/10.1073/pnas.96.6.2896.

PERDIGÃO, J.; SILVA, H.; MACHADO, D. et al. (2014). Unraveling Mycobacterium tuberculosis genomic diversity and evolution in Lisbon, Portugal, a highly drug resistant setting. BMC Genomics. v. 18, p. 991. https://doi.org/10.1186/1471-2164-15-991

PHELAN, J. E.; COLL, F.; BERGVAL, I. et al. (2016). Recombination in pe/ppe genes contributes to genetic variation in Mycobacterium tuberculosis lineages. BMC Genomics. v. 17, n. 151. https://doi.org/10.1186/s12864-016-2467-y

PITASSI, C.; GONÇALVES, A. A.; MORENO-JUNIOR, V. A. (2014). Fatores que influenciam a adoção de ferramentas de TIC nos experimentos de bioinformática de organizações biofarmacêuticas. Ciências \& Saúde Coletiva, v. $19, \quad$ n. 01. https://doi.org/10.1590/1413-81232014191.2007

RAMAKRISHNAN, L. (2000). Granuloma-specific expression of Mycobacterium virulence proteins from the glycine-rich PE-PGRS family. Science. v. 288, n. 5470, p. 1436-1439. https://doi.org/10.1126/science.288.5470.1436 
REED, S. G.; COLER, R. N.; DALEMANS, W. et al. (2009). Defined tuberculosis vaccine, Mtb72F/AS02A, evidence of protection in cynomolgus monkeys. Proceedings of The National Academy of Sciences. v. 106, n. 7, p. 2301-2306. https://doi.org/10.1073/pnas.0712077106

SILVA, D. R.; MELlO, F. C. Q.; MIGLIORI, G. B. (2020). Esquemas mais curtos de tratamento da tuberculose: o que há de novo? Journal Brasileiro de Pneumologia, v. 46, n. 2:e20200009. http://dx.doi.org/10.36416/1806-3756/e20200009

STRONG, M.; SAWAYA, M. R.; WANG, S. et al. (2006). Toward the structural genomics of complexes: Crystal structure of a PE/PPE protein complex from Mycobacterium tuberculosis. PNAS. v. 103, n. 21, p. 8060-8065. https://doi.org/10.1073/pnas.0602606103

STURKENBOOM, M. G. G.; SIMBAR, N.; AKKERMAN, O. W. et al. (2018). Amikacin Dosing for MDR tuberculosis: a systematic review to establish or revise the current recommended dose for tuberculosis treatment. Clinical Infectious Diseases. v. 67, n. 3, p. 303307. https://doi.org/10.1093/cid/ciy613

TUNDUP, S.; MOHAREER, K.; HASNAIN, S. E. (2014). Mycobacterium tuberculosis PE25/PPE41 protein complex induces necrosis in macrophages: Role in virulence and disease reactivation? Febs Open Bio. v. 4, n. 1, p. 822-828. https://doi.org/10.1016/j.fob.2014.09.001

VILLAS, J. L; TABASSUM, N.; MOTA, J. et al. (2018). Advances in image processing for single-particle analysis by electron cryomicroscopy and challenges ahead. Current Opinion in Structural Biology. v. 52, p. 127-145. https://doi.org/10.1016/j.sbi.2018.11.004

WHO. World Health Organization. (2019). Global tuberculosis report 2019. Geneva: World Health Organization.

WOODWORTH, J. S.; COHEN, S. B.; MOGUCHE, A.O. et al. (2016). Subunit vaccine H56/CAF01 induces a population of circulating CD4 T cells that traffic into the Mycobacterium tuberculosis-infected lung. Mucosal Immunology. v. 10, n. 2, p. 555-564. https://doi.org/10.1038/mi.2016.70 